J3eA, Journal sur l'enseignement des sciences et technologies de l'information et des systèmes, Volume 2, Hors-Série 2, 13 (2003)

DOI : http://dx.doi.org/10.1051/bib-j3ea:2003613

(C) EDP Sciences, 2003

\title{
La CEM au sein des dispositifs électroniques intégrés
}

D. Magnon

Laboratoire de Microélectronique de Puissance

16 rue Pierre et Marie Curie

BP 7155

F-37071 Tours Cedex 2, France

magnoneuniv-tours. fr 


\section{La CEM au sein des dispositifs électroniques intégrés.}

\author{
Didier MAGNON \\ Université de Tours \\ Laboratoire de Microélectronique de Puissance \\ 16, rue Pierre et Marie Curie \\ BP 7155 \\ 37071 Tours CEDEX 2 \\ France
}

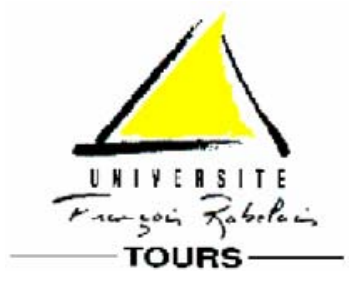

E-mail : .magnoneuniv-tours.fr

\section{Résumé}

Ces dernières années, l'évolution des technologies de la microélectronique a permis la conception de plusieurs composants de puissance sur le même monocristal. Ceci conduit tout naturellement à la réalisation de fonctions plus ou moins complexes sur la même puce ou sur plusieurs puces réunies dans le même boîtier. Les avantages sont considérables, puisque nous obtenons, entre autres, des réductions de volume et de coût de fabrication. Lla mise au point des systèmes est quasiment réduite et la Compatibilité ElectroMagnétique (CEM), susceptibilité et émission, est nettement améliorée. C'est ce dernier point, au travers d'exemples de fonctions intégrées de puissance, que nous nous proposons de traiter ici. Nous montrons notamment comment réaliser un variateur de lumière sans filtre et comment prendre en compte les normes CEM dans la réalisation physique des composants de puissance.

\section{Introduction}

Intégrer sur une même puce plusieurs composants de puissance pour réaliser une fonction est aujourd'hui une réalité industrielle. Historiquement, la première approche d'une intégration de composants de puissance a été la "smart power". Par la suite une seconde approche a vu le jour, ses inventeurs la nomment "ASD ${ }^{\mathrm{TM}}$ " (Application Specific Device) [1]. Les applications concernées par cette dernière sont aussi bien dans le domaine industriel (convertisseur statique, variateur de vitesse, ...) que dans le domaine domestique. La smart power est schématiquement composée d'un interrupteur de puissance et de sa commande sur la même puce. Elle est principalement utilisée en basse tension DC, typiquement dans l'automobile (régulation d'alternateur, injection, ....)[2]. Les $\mathrm{ASD}^{\mathrm{TM}}$ ne rassemblent que les composants de puissance, la commande est à l'extérieur. On les utilise en haute tension (DC ou AC). On les rencontre principalement dans les applications $240 \mathrm{~V} / 50 \mathrm{~Hz}$ dont la consommation varie de quelques $\mathrm{mA}$ à environ 50A [3], [4]. les deux approches sont donc complémentaires.

\section{Améliorations de fonctions intégrées}

Depuis 1996, la CEM est devenue un challenge important pour les concepteurs d'alimentations, qu'elles soient à découpage ou non. En effet, ils doivent se préoccuper de deux points pour leurs systèmes :

$\square$ éviter qu'ils soient une source de perturbation,

$\square$ faire en sorte qu'ils soient immunisés vis-à-vis des interférences électromagnétiques extérieures.

Les applications concernées par les fonctions de puissance intégrées que nous présentons ici sont les gradateurs monophasés et les commandes d'électrovannes ou de ventilateurs des produits blancs (machine à laver).

Lorsque l'on regarde la structure actuelle des gradateurs domestiques, on se rend vite compte que les concepteurs réalisent de la CEM curative. En effet, la présence d'un filtre plus volumineux et aussi onéreux que la fonction ellemême atteste cette affirmation. Nous avons donc voulu faire de la CEM conceptuelle afin de voir s'il était possible de limiter, voire de supprimer ce filtre. Cette étude nous a conduits à la modification de la structure même des fonctions intégrées.

\subsection{Cellule ASDTM à GTO}

La cellule $\mathrm{ASD}^{\mathrm{TM}}$ à base de GTO que nous avons étudiée est représentée en gris sur la Figure 1.

L'ensemble constitue un hacheur secteur fonctionnant selon le mode thyristor dual. La partie grisée est intégrable.

La charge voit une partie de la sinusoïde secteur pendant une durée qui dépend du pont diviseur réalisé par R1 et R2 et de la diode zener D1 : il s'agit d'une commande à angle de phase constant. 


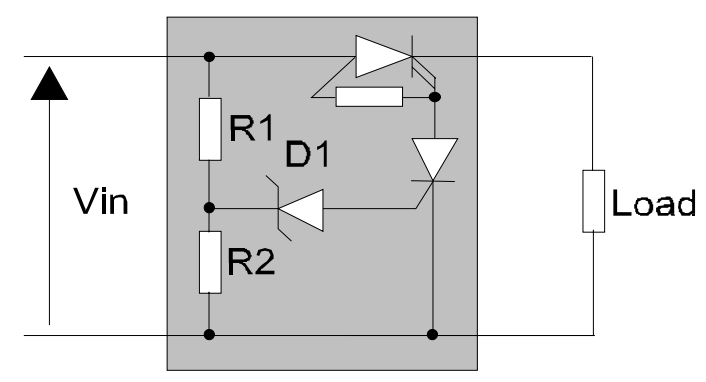

Figure 1 : hacheur secteur utilisant une fonction intégrée à base de GTO. La commutation se fait sur charge résistive. La partie grisée est intégrable

Lorsque l'on effectue une mesure CEM, selon la norme NF EN55014 [5], sur ce montage, on obtient (Figure 2) le spectre des modes commun et différentiel des perturbations en émission conduite. Ce spectre est comparé au gabarit de la norme.

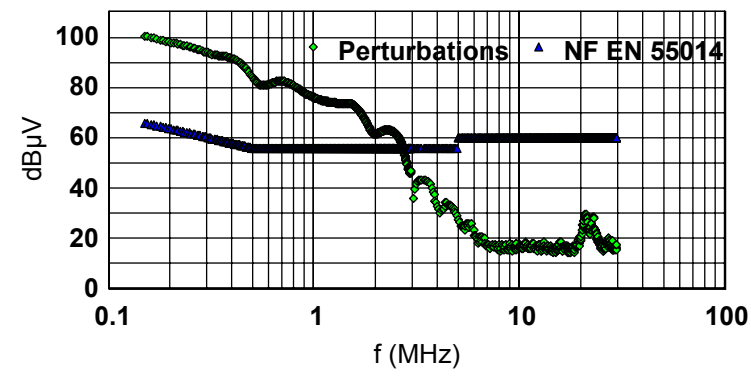

Figure 2 : perturbations conduites générées par le montage de la Figure 1. Le courant coupé est de $1 \mathrm{~A}$

Cette mesure montre clairement, alors que le courant coupé n'est que de $1 \mathrm{~A}$, le dépassement des perturbations jusqu'à environ $3 \mathrm{MHz}$ (plus $35 \mathrm{~dB}$ à $150 \mathrm{kHz}$ ). Il est donc impératif d'agir.

Notre étude s'est alors confinée à la recherche des phénomènes perturbateurs dans une onde secteur dont la fréquence de hachage est comprise entre $50 \mathrm{~Hz}$ et $5 \mathrm{kHz}$ [6]. Les résultats de cette étude ont montré que les perturbations sont générées non pas par les forts $\mathrm{di} / \mathrm{dt}$ ou $\mathrm{dv} / \mathrm{dt}$, mais par les points de raccordement entre deux segments [7], [8], [9].

Parmi les formes de commutation les plus performantes, nous avons choisi la sinusoïde car elle peut être réalisée avec quelques composants.

Remarque : Le domaine dans lequel nous travaillons nous impose des coûts les plus bas possibles, donc l'ajout d'un microcontrôleur est impossible. De surcroît, une forme élaborée de façon numérique présente des sauts de quantification. Avec seulement 8 bits, le signal numérique lui même serait perturbateur.

En fait, réaliser une commutation sinusoïdale revient à allonger le temps de commutation et à faire travailler le composant de puissance en linéaire. Cette constatation nous a conduits à élaborer le circuit de la Figure 3 [10].

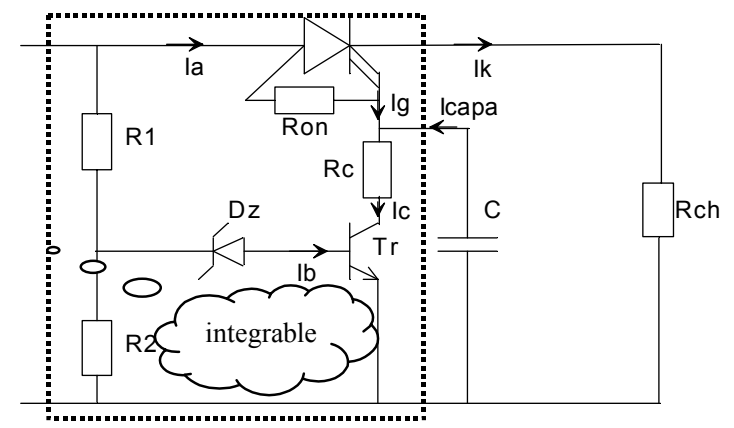

Figure 3 : schéma de la nouvelle structure.

La commutation obtenue est représentée sur la Figure 4.

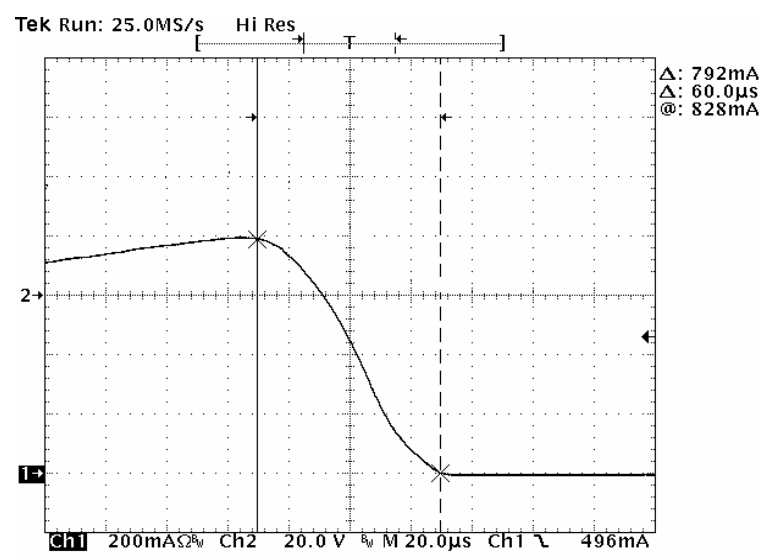

Figure 4 : commutation de la fonction de la Figure 3.

La mesure des perturbations électromagnétiques du nouveau schéma est portée sur la Figure 5.

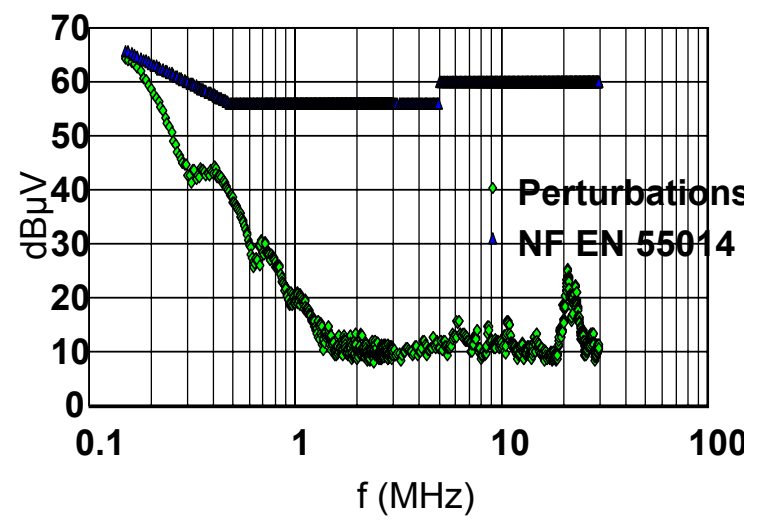

Figure 5: spectre des perturbations conduites de la fonction modifiée

Notre hacheur secteur est maintenant compatible avec la norme NF EN55014, sans aucun filtre secteur. Néanmoins, cette fonction, en boîtier DIL, est très limitée en puissance de sortie $(<100 \mathrm{~W})$. Nous nous sommes donc intéressés à des interrupteurs plus puissants, les "Mos Bi-directionnels switches" (MBS) et les "Insulated Gate THyristor" (IGTH). Les études étant proches, nous présenterons, ici, uniquement les résultats de l'IGTH.

Un IGTH est une association entre un IGBT en entrée et un thyristor en sortie [11]. La Figure 6 montre la structure d'un tel composant. 


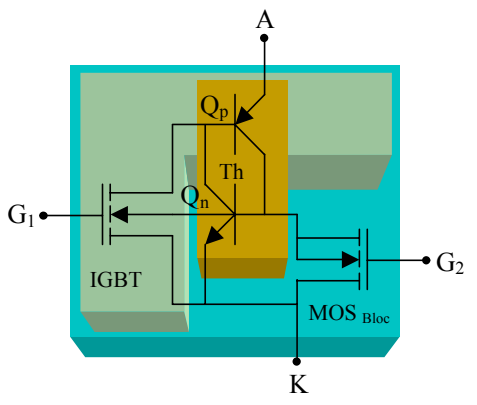

Figure 6 : Schéma équivalent d'un IGTH intégré

Pour fermer un IGTH, il faut d'abord commander l'IGBT, puis quand ce dernier est conducteur, on enclenche le thyristor. L'IGBT est alors ouvert; c'est le thyristor qui supporte la puissance. L'ouverture se fait de façon naturelle, lorsque le courant qui traverse l'IGTH devient inférieur au courant de maintien du thyristor (Ih). Avec cette structure on peut contrôler la mise en conduction de l'interrupteur.

Pour notre étude, nous avons réalisé un IGTH en discret de manière à pouvoir modifier les composants à notre guise. Le schéma utilisé est celui de la Figure 7.

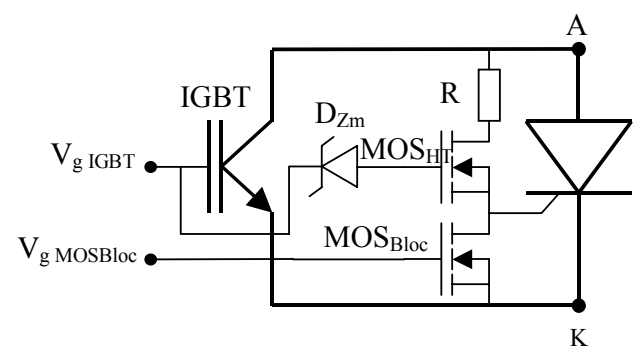

Figure 7 : Structure discrète d'un IGTH

Si on réalise un gradateur avec une telle structure, et que l'on applique une commande carrée, on obtient là encore une mesure spectrale similaire à celle de la Figure 2.

Afin d'obtenir une commutation sinusoïdale, nous avons ajouté à notre structure, deux impédances : $Z_{G}$ et $Z_{\text {MILLER }}$ (Figure 8) pour agir respectivement sur le début et sur la fin de la commutation [12].

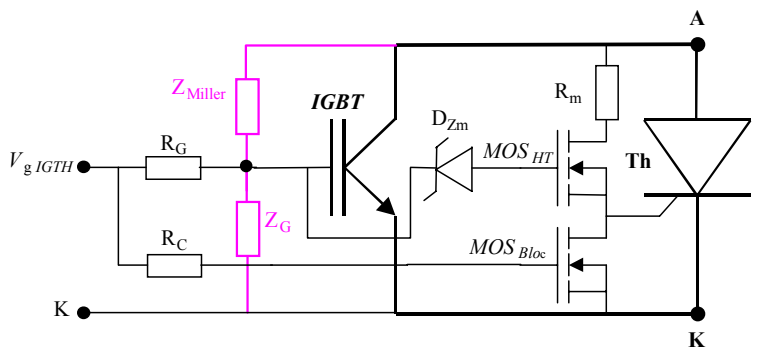

Figure $8: Z_{G}$ et $Z_{\text {MILLER }}$ sont ajoutées pour réaliser une commutation sinusoïdale

Les meilleurs résultats ont été obtenus avec une capacité en $Z_{\mathrm{G}}$ et la capacité d'une diode en $Z_{\text {MILLER (Figure 9). Les }}$ diodes zener servent d'une part à compenser la tension de seuil et d'autre part à saturer correctement l'IGBT.

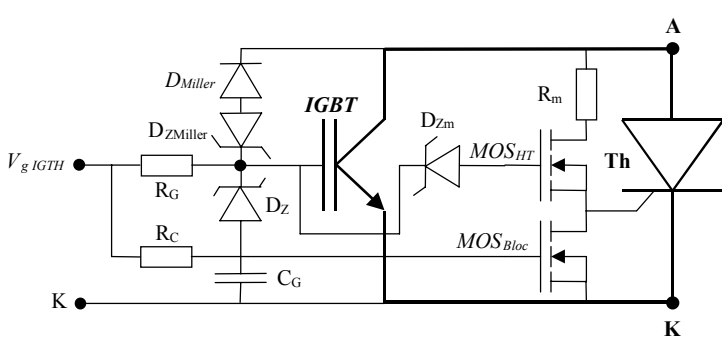

Figure 9 : interface permettant une commutation sinusoïdale

Une comparaison entre une commutation sinusoïdale idéale et la commutation réelle est portée sur la Figure 10.

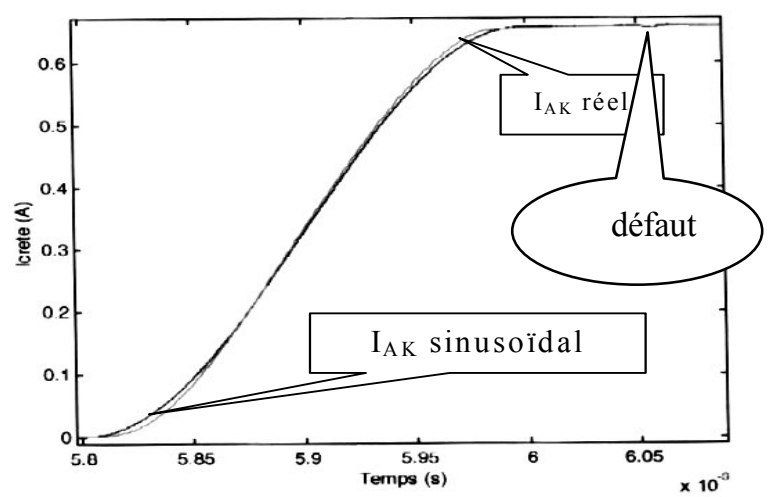

Figure 10 : Comparaison entre une sinusoïde parfaite et la commutation réelle à la fermeture de I'IGTH

L'erreur entre les deux courbes est inférieure à $0,4 \%$. Cependant, un défaut à peine visible (Figure 10) existe sur la commutation réelle. Il s'agit d'un saut de courant qui est dû au "passage" IGBT/thyristor. En effet, ces deux composants ont des tensions de déchet différentes, ce qui génère cette variation de courant. Bien que cela paraisse bénin, ce saut de courant fait perdre presque $25 \mathrm{~dB}$ [12].

Les designers devront donc réaliser des composants IGBT et thyristors avec des tensions de déchet proches. Nous avons également relevé un problème, à une échelle moindre, identique lors de l'ouverture naturelle du thyristor. Si le Ih est élevé, il génère d'importantes perturbations qui peuvent à l'extrême, rendre l'application non conforme.

Avec cette fonction, l'application est CEM sans filtre jusqu'à $150 \mathrm{~W}$. Au-delà, le saut de courant précédemment évoqué ne permet pas d'atteindre notre objectif.

Cela étant, nous avons élaboré des fonctions de puissance entièrement intégrables et qui sont compatibles CEM sans aucun filtre secteur.

\subsection{Amélioration de l'immunité}

Parmi les différentes fonctions $\mathrm{ASD}^{\mathrm{TM}}$ que nous avons étudiées, il en existe qui sont composées de plusieurs fonctions identiques sur la même puce. Par exemple, l'ACS 402 contient 4 interrupteurs alternatifs en parallèle (Figure 11). Chaque interrupteur est équivalent à un triac protégé. 
Les 4 ACS possèdent une commande indépendante mais une anode en commun

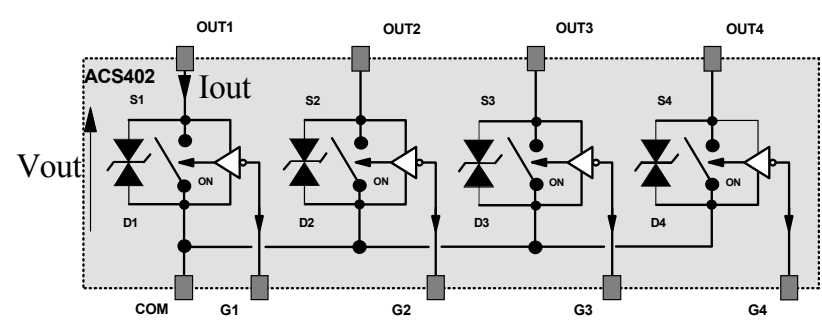

Figure 11 : Structure d'un ACS402

Ces composants sont destinés à piloter des électrovannes ou des ventilateurs, c'est-à-dire des charges qui fonctionnent en pleine onde. Il est donc inutile d'appliquer les normes précédentes. Par contre, le fonctionnement d'un interrupteur ne doit pas être modifié par un autre ou par une perturbation extérieure. On applique donc les normes d'immunité. Nous allons voir ici l'influence des ondes de la norme qui reproduit les décharges électrostatiques IEC 1000-4-4 (Figure 12) [13].

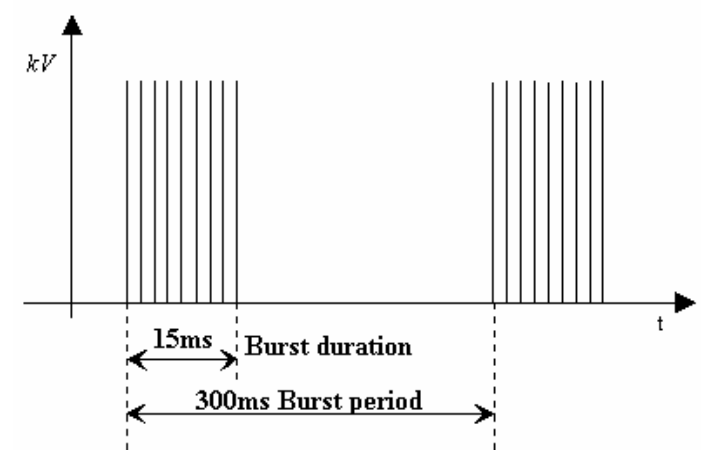

Figure 12 : Salves de la norme IEC1000-4-4.

L'amplitude des salves peut aller jusqu'à $4 \mathrm{kV}$.

Nous avons soumis 3 lots d'ACS402 aux salves de cette norme. Ces trois lots concernent des ACS qui, bien qu'ayant la même fonction, ont une structure différente [14].

Les tests sont effectués avec :
$\square$ la gâchette ouverte,
$\square \quad$ une polarité positive des impulsions
$\square \quad$ Vout $>0$; la tension secteur plus les impulsions sont connectées sur la borne Vout,
$\square$ Vout $<0$ on inverse les connexions par rapport à Vout $>0$.

Au-delà de $1000 \mathrm{~V}$, les trois structures entrent en conduction bien qu'il n'y ait aucun signal sur la gâchette (Table 1).

\begin{tabular}{|c|c|c|c|c|}
\hline Voltage & \multicolumn{1}{|l|}{$\begin{array}{l}\text { signe de } \\
\text { Vout }\end{array}$} & batch 1 & batch 2 & batch 3 \\
\hline 700 & $>0$ & $\mathrm{NE}$ & $\mathrm{NE}$ & $\mathrm{E}$ \\
\cline { 2 - 5 } & $<0$ & $\mathrm{NE}$ & $\mathrm{NE}$ & $\mathrm{E}$ \\
\hline 1000 & $>0$ & $\mathrm{E}$ & $\mathrm{NE}$ & $\mathrm{E}$ \\
\hline
\end{tabular}

\begin{tabular}{|c|c|c|c|c|}
\hline & $<0$ & $\mathrm{E}$ & $\mathrm{NE}$ & $\mathrm{E}$ \\
\hline \multirow{2}{*}{2000} & $>0$ & $\mathrm{E}$ & $\mathrm{E}$ & $\mathrm{E}$ \\
\cline { 2 - 5 } & $>0$ & $\mathrm{E}$ & $\mathrm{E}$ & $\mathrm{E}$ \\
\hline
\end{tabular}

Table 1: Résultats des tests d'immunité sur 3 lots d'ACS402. NE= non enclenché, $\mathrm{N}=$ enclenché

Bien évidemment ce test n'est pas du tout satisfaisant. Après analyse des résultats, les designers ont déduit plusieurs règles de conception. Ils ont alors réalisé deux nouveaux design: l'ACS 402 et l'ACS 108. Les tests normatifs ont à nouveau été appliqués à ces composants. Les résultats sont consignés dans la Table 2.

\begin{tabular}{|c|c|c|c|}
\hline \multirow{2}{*}{ Voltage } & $\begin{array}{l}\text { Signe de } \\
\text { Vout }\end{array}$ & $\begin{array}{c}\text { ACS108- } \\
\text { 5SA }\end{array}$ & $\begin{array}{c}\text { ACS402- } \\
\text { 5SB4 }\end{array}$ \\
\hline \multirow{2}{*}{500} & $>0$ & $\mathrm{NE}$ & $\mathrm{NE}$ \\
\cline { 2 - 4 } & $<0$ & $\mathrm{NE}$ & $\mathrm{NE}$ \\
\hline \multirow{2}{*}{1000} & $>0$ & $\mathrm{NE}$ & $\mathrm{NE}$ \\
\cline { 2 - 4 } & $<0$ & $\mathrm{NE}$ & $\mathrm{NE}$ \\
\hline \multirow{2}{*}{2000} & $>0$ & $\mathrm{NE}$ & $\mathrm{NE}$ \\
\cline { 2 - 4 } & $<0$ & $\mathrm{NE}$ & $\mathrm{NE}$ \\
\hline
\end{tabular}

Table 2 : résultats des tests d'immunité sur deux lots d'ACS modifiés. NE= non enclenché

Nous pouvons voir que cette fois-ci il n'y a aucun amorçage quel que soit l'ACS. Par conséquent, ceci montre qu'il est possible d'améliorer le comportement des fonctions intégrées lors de leur phase de fabrication.

\section{Conclusion}

L'intégration des composants de puissance sur une même puce est aujourd'hui possible grâce à la smart power ou aux $\mathrm{ASD}^{\mathrm{TM}}$. Ces derniers permettent l'association de composants MOS et bipolaires.

La technologie $\mathrm{ASD}^{\mathrm{TM}}$ intègre de plus en plus de composants grâce aux performances croissantes de la lithographie notamment.

Nous avons dans cet article mis en évidence la pollution électromagnétique des fonctions intégrées. Nous avons étudié l'émission électromagnétique pour les unes et leurs réactions aux agressions d'un environnement hostile pour les autres.

Nous proposons quelques modifications simples du design des fonctions intégrées pour qu'elles présentent des perturbations aux limites des valeurs autorisées. Les résultats de notre étude de susceptibilité ont permis de redessiner la périphérie d'un ACS constitué de plusieurs interrupteurs à anode commune. Au final, ces nouveaux ACS supportent des ESD supérieures à $2000 \mathrm{~V}$.

Nous disposons donc :

$\square$ de plusieurs fonctions intégrées de puissance dont les perturbations en mode conduit sont en accord, sans filtre secteur, avec les limites de la norme NF EN55014. Les applications principales sont les 
gradateurs de lumière et les alimentations $\mathrm{AC} / \mathrm{DC}$ faible puissance sans transformateur.

$\square \quad$ d'interrupteurs en parallèle, avec anode commune, qui sont capables de travailler dans un environnement électromagnétique décrit par la norme IEC 1000-4-4.

Grâce à ces fonctions intégrées, nos applications présentent un volume réduit et elles sont moins onéreuses. De plus, elles ne nécessitent pas de filtre ou de protection particulière.

Par conséquent, l'amélioration des fonctions intégrées passe obligatoirement par des avancées technologiques, mais également par la prise en compte, dès la conception, des contraintes dues aux normes CEM.

\section{Références}

[1] J.B. QUOIRIN, R. PEZZANI. "Intégration monolithique, état de l'art et tendance future" Forum Européen des semi-conducteurs de puissance. Club CRIN - SEE, 1997, pp. 9-14.

[2] "Designers' guide to power products - Application manual". 2nd edition SGS Thomson Microelectronics, 1992.

[3] R. PEZZANI, J.B. QUOIRIN. "Functional integration of power devices : a new approach". EPE'95, Sevilla, 1995, pp. 2.219-2.223.

[4] J.L. SANCHEZ, P. AUSTIN, R. BERRIANE, M. MARMOUGET. "Trends in design and technology for new power integrated devices based on functional integration". EPE'97, Trondheim, 1997, pp. 1.302-1.307.

[5] NF EN 55014, "Limites et méthodes de mesure des perturbations radioélectriques produites par les appareils électrodomestiques ou analogues comportant des moteurs ou des dispositifs thermiques, par les outils électriques et par les appareils électriques analogues”, UTE, 1993.

[6] F. GUITTON :"Etude des formes d'onde CEM permettant d'éliminer le filtre secteur d'un convertisseur commutant des charges résistives directement sur le réseau basse tension. Application aux circuits intégrés de puissance ASD ${ }^{\mathrm{TM}}$ ". Thèse de doctorat de l'université de Tours, Octobre 1999.

[7] F. GUITTON, D. MAGNON : "Utilisation en mode linéaire d'un GTO "normally-on" pour commuter sur le $240 \mathrm{~V} / 50 \mathrm{~Hz}$ des puissances inférieures à $1 \mathrm{~kW}$ ", Proceeding EPF'98,, Belfort, 16-18 decembre 98, pp. 3540.

[8] F. GUITTON, D. MAGNON : "Determination of a relevant criterion to characterize differential conducted perturbations generated by low frequency converters", Revue IEEE trans. Commun., vol.E83-B, march 2000.
[9] F. GUITTON, D. MAGNON : "Détermination des $\mathrm{di} / \mathrm{dt}$ permettant à un interrupteur solide de commuter le secteur en respectant les normes CEM conduites", Proceeding EPF'98, Belfort, 16-18 decembre 98, pp. 225228.

[10] F. GUITTON, D. MAGNON, J.M. SIMONNET, O. LADIRAY : "Circuit de commutation", Demande de BREVET D'INVENTION N 98/10929, 28 août 98.

[11] JALADE J., SANCHEZ J.-L., LAUR J.-P., BREIL M., AUSTIN P., BERNIER E., ROY M.; Brevet d'invention :Interrupteur de puissance à di/dt contrôlé; déposant : STMicroelectronics SA; Numéro : 98/16771

[12] N. NOIROT, D. MAGNON, "La fonction intégrée de puissance IGTH et la CEM", CEMEXPO'01, Paris, octobre 02 .

[13] IEC 1000-4-4, "Electromagnetic Compatibility (EMC) Part 4: Testing and measurement techniques. Section 4: Electrical fast transient / burst immunity test" Basis EMC Publication. Editor, Union Technique de l'Université, June 95.

[14] D. MAGNON, A. FEYBESSE, F. GUITTON : "Burst immunity tests according to IEC 1000-4-4 standard use to improve design of a new integrated power function", Proceeding EMC'99, IEEE, Seattle, USA, 2-6 August 99 